ACCEPTED TO APJL

Preprint typeset using $\mathrm{LAT}_{\mathrm{E}} \mathrm{X}$ style emulateapj v. 5/2/11

\title{
A HIGH-VELOCITY BULGE RR LYRAE VARIABLE ON A HALO-LIKE ORBIT
}

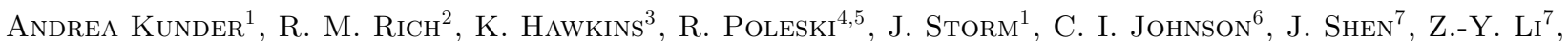 \\ M. J. Cordero ${ }^{8}$, D. M. NATAF ${ }^{9}$, G. Bono ${ }^{10,11}$, A. R. WAlker ${ }^{12}$, A. Koch ${ }^{13}$, R. De Propris ${ }^{14}$, A. Udalski $^{5}$, \\ M. K. Szymański ${ }^{5}$, I. Soszyński ${ }^{5}$, G. Pietrzyński ${ }^{5,15}$, K. UlaczyK ${ }^{5,16}$, Ł. Wyrzykowski ${ }^{5}$, P. Pietrukowicz ${ }^{5}$, J.

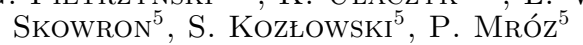 \\ accepted to ApJL
}

\begin{abstract}
We report on the RR Lyrae variable star, MACHO 176.18833.411, located toward the Galactic bulge and observed within the data from the ongoing Bulge RR Lyrae Radial Velocity Assay (BRAVA-RR), which has the unusual radial velocity of $-372 \pm 8 \mathrm{~km} \mathrm{~s}^{-1}$ and true space velocity of $-482 \pm 22 \mathrm{~km} \mathrm{~s}$ relative to the Galactic rest frame. Located less than $1 \mathrm{kpc}$ from the Galactic center and toward a field at $(l, b)=(3,-2.5)$, this pulsating star has properties suggesting it belongs to the bulge RR Lyrae star population yet a velocity indicating it is abnormal, at least with respect to bulge giants and red clump stars. We show that this star is most likely a halo interloper and therefore suggest that halo contamination is not insignificant when studying metal-poor stars found within the bulge area, even for stars within $1 \mathrm{kpc}$ of the Galactic center. We discuss the possibility that MACHO 176.18833.411 is on the extreme edge of the bulge RR Lyrae radial velocity distribution, and also consider a more exotic scenario in which it is a runaway star moving through the Galaxy.
\end{abstract}

Subject headings: Galaxy: bulge; Galaxy: kinematics and dynamics; Galaxy: structure; surveys

\section{INTRODUCTION}

It is well known that within the Galactic bulge at longitudes $|l|<10^{\circ}$, there is a bar-like structure with a bar angle in the range $20-30^{\circ}$, traced by numerous old stellar population probes (e.g., red clump giants, Stanek 1994, Miras, Groenewegen \& Blommaert 2005). This bar/bulge is a rotating Box/Peanut $(\mathrm{B} / \mathrm{P})$ structure with a X-shape protrusion, made up largely of old and metalrich stars $(\sim 10 \mathrm{Gyr},[\mathrm{Fe} / \mathrm{H}]$ falling between -0.5 and +0.5 dex, e.g., Johnson et al. 2013; Gonzalez et al. 2012;

\footnotetext{
${ }^{1}$ Leibniz-Institut für Astrophysik Potsdam (AIP), An der Sternwarte 16, D-14482 Potsdam, Germany

2 Department of Physics and Astronomy, University of California at Los Angeles, Los Angeles, CA 90095-1562, USA

3 Institute of Astronomy, Madingley Road, Cambridge CB3 OHA, UK

4 Department of Astronomy, Ohio State University, $140 \mathrm{~W}$. 18th Ave., Columbus, OH 43210, USA

${ }^{5}$ Warsaw University Observatory, Al. Ujazdowskie 4, 00-478 Warszawa, Poland

${ }^{6}$ Harvard-Smithsonian Center for Astrophysics, Cambridge, MA 02138

${ }^{7}$ Key Laboratory for Research in Galaxies and Cosmology, Shanghai Astronomical Observatory, Chinese Academy of Sciences, 80 Nandan Road, Shanghai 200030, China

8 Astronomisches Rechen-Institut: Zentrum für Astronomie, Mnchhofstr. 12-14, 69120 Heidelberg Germany

9 Research School of Astronomy and Astrophysics, The Australian National University, Canberra, ACT 2611, Australia

10 Dipartimento di Fisica, Universita di Roma Tor Vergata, vi a Della Ricerca Scientifica 1, 00133, Roma, Italy

${ }^{11}$ INAF, Rome Astronomical Observatory, via Frascati 33 00040, Monte Porzio Catone, Italy

12 Cerro Tololo Inter-American Observatory, National Optical Astronomy Observatory, Casilla 603, La Serena, Chile

${ }^{13}$ Landessternwarte, Zentrum für Astronomie der Universität

Heidelberg, Königstuhl 12, D-69117 Heidelberg, Germany

${ }^{14}$ Finnish Centre for Astronomy with ESO (FINCA), University of Turku, Finland

${ }^{15}$ Universidad de Concepción, Departamento de Astronomia, Casilla 160-C, Concepción, Chile

16 Department of Physics, University of Warwick, Gibbet Hill Road, Coventry, CV4 7AL, UK
}

Wegg \& Gerhard 2013).

There is speculation that the Milky Way also has an older, more spheroidal bulge population, and perhaps the greatest possibility of uncovering such a component would be within the most metal-poor bulge stars. One example of a metal-poor bulge population is the RR Lyrae stars (RRLs), and over the past few years, $\sim 38000$ RRLs toward the bulge have been identified from photometric surveys (e.g., Soszyński et al. 2014). These RRLs are thought to exhibit a small metallicity spread and are centered around $[\mathrm{Fe} / \mathrm{H}]=-1$ dex (Walker \& Terndrup 1991: Kunder \& Chaboyer 2008, Soszyński et al. 2014). The small $[\mathrm{Fe} / \mathrm{H}]$ spread may suggest that RRLs trace a more ancient stellar population than the majority of bulge RGB and red clump stars, which are more metalrich, on average, and likely do not evolve to become RRLs (e.g. Lee 1992: Walker \& Terndrup 1991).

Recent photometric studies have reached different conclusions regarding the relationship of the bulge RRLs and the bar/bulge. Dékány et al. (2013) combined optical and infrared photometry of $\sim 8000$ ss OGLE-III discovered RRLs to find that unlike the red clump giants (RCGs), RRLs do not trace a strong bar. Instead, they have a more spheroidal, centrally concentrated distribution, indicating that the RRLs belong to a classical bulge that has co-evolved with the bar (e.g., Saha \& Gerhard 2013).

In contrast, using 28000 RRLs from the more spatially extended OGLE-IV bulge sample, Pietrukowicz et al. (2014) assert that the RRLs trace closely the barred structure formed of RCGs, and hence that the bulge RRLs are in the same gravitational potential together with the more massive Galactic bar.

Missing still are the kinematics of the bulge RRLs, which can resolve this discrepancy and provide an understanding of the origin of the old, metal-poor bulge component. The last published paper on bulge RRLs radial velocities, Gratton (1987), used a sample of 17 
RRLs to conclude that the kinematic properties of RRLs in Baade's Window are similar to that of the Miras, Mgiants, K-giants, $\mathrm{OH} / \mathrm{IR}$ sources and planetary nebulae of the Galactic bulge.

In this paper, we report on a high-velocity RRL found serendipitously in the Bulge RR Lyrae Radial Velocity Assay (BRAVA-RR) to have $v_{r}=-372 \mathrm{~km} \mathrm{~s}^{-1}$, which is well above the typical speed of the stars one might expect to find in the bulge. High-velocity stars are intriguing in part because they can provide insight to the mechanisms that produce their velocities. The origin of high-velocity stars can also provide useful information about the environments from which they are produced. Here we investigate the cause for the high-velocity of MACHO 176.18833.411 to discern whether it is consistent with stars in the Galactic bulge and what this suggests about the formation of the Galaxy.

\section{OBSERVATIONS AND RADIAL VELOCITY}

MACHO 176.18833 .411 was originally catalogued by the MACHO survey as a fundamental mode RRL (Kunder et al. 2008 ${ }^{17}$. The star was one of the $\sim 100$ RRLs surveyed spectroscopically as part of BRAVA-RR (NOAO PropID: 2014A-0143; PI: A. Kunder) in a field at $(l, b)=(3,-2.5)$ using the AAOmega multifiber spectrograph on the Anglo-Australian Telescope (AAT). We observed this star twice on June 21, 2014, separated in time by 7 hours, and the observations were taken in dual beam mode centered on $8600 \AA$, with the $580 \mathrm{~V}$ and $1700 \mathrm{D}$ gratings to probe the Calcium Triplet. This covers the optical window from about $8300 \AA$ to $8800 \AA$ at a resolution of $\mathrm{R} \sim 10,000$.

The data were reduced using the automated pipeline supplied by AAOmega, 2DFDR, and the spectra were cross-correlated using the IRAF cross-correlation routine, xcsoa. Four Bulge RAdial Velocity Assay (BRAVA) stars (Kunder et al. 2012) observed with the same setup were selected as radial velocity standard stars. Due to less than optimal weather conditions, the signal-to-noise ratio is $\sim 10$ (see spectrum in Figure 1), and the consistency of our velocity result is $8 \mathrm{~km} \mathrm{~s}^{-1}$, in agreement with the errors reported by xcsao for each individual measurement.

Figure 1 (middle) shows the pulsation curve using the radial velocity template and scaled by its $V$-amplitude as outlined from Liu (1991). The two radial velocity measurements were folded by the known period to find the radial velocity as a function of phase. Both measurements fit the radial velocity template well for a RRL with a line-of-sight radial velocity, $V_{\text {los }}$ of $-372 \mathrm{~km} \mathrm{~s}^{-1}$. It is noteworthy that we did not adjust the template in phase, which indicates that the OGLE time of maximum brightness is reliable for this star.

\section{THE PROPERTIES OF MACHO 176.18833.411}

The OGLE-IV catalogue of RRLs provides $V$ - and $I$ band light curves of their stars, with the photometric observations spanning from March 2010 to October 2013 (Soszyński et al. 2014). The optical light curve for MACHO 176.18833.411 from the OGLE-IV observations is shown in Figure 1 (left), and its most important properties are summarised in Table 1.

17 This RRL is also designated OGLE-BLG-RRLYR-10353.
To find the distance to the RRL, we first use the meanflux magnitude as listed by OGLE-IV. Next, the $\mathrm{E}(V-I)$ color excess along the stars line of sight is calculated from the observed $V-I$ color at minimum light, $(V-I)_{\text {min,obs }}$ (see e.g., Guldenschuh et al. 2005), by carrying out a Fourier fit to the $V$ and 1 -band light-curves.

The extinction, $A_{I}$ can then be derived using:

$$
A_{I}=0.7465 E(V-I)+1.37 E(J-K),
$$

as introduced in Nataf et al. (2013). Here, E $(J-K)$ was taken from the bulge reddening maps of Gonzalez et al. (2012). Finally, it has been shown that RRLs $V$-band light curves and the phase difference of their Fourier decomposition can be used to estimate photometric metallicities to an accuracy of $0.2 \operatorname{dex}($ Jurcsik \& Kovács 1996), although with high-quality light curves, the fitting accuracy can be $\sim 0.12$ dex (Kovács 2005). This relation is well-calibrated over the metallicity range from $\sim-2.0$ dex to $\sim 0$ dex. Applying this method to the OGLE $V$-band light curve, the metallicity $([\mathrm{Fe} / \mathrm{H}])$ of MACHO 176.18833.411 is estimated and placed on the Carretta et al. (2009) metallicity scale. Using the recalibration of the RRL luminosity scale by Catelan \& Cortés (2008), MACHO 176.18833.411 has an absolute magnitude of $M_{V}=0.58 \pm 0.13$ mag. Similarly, using a quadratic relation between RRLs absolute magnitude and metallicity from Bono, Caputo, \& di Criscienzo (2007), we find $M_{V}=0.61 \pm 0.08 \mathrm{mag}$, where 0.08 is a reasonable error in the RRLs absolute magnitude- $[\mathrm{Fe} / \mathrm{H}]$ zero-point calibration. The Benedict et al. (2011) $M_{V}-[\mathrm{Fe} / \mathrm{H}]$ relation, however, results in $M_{V}=0.43 \pm 0.07$, indicating that the level of agreement between independent $M_{V}$ measurements is as large as $\sim 0.2 \mathrm{mag}$. A systematic uncertainty of $0.2 \mathrm{mag} M_{V}$ is factored into the uncertainty in the estimated distance, and does not change the results significantly. The apparent magnitude, reddening and absolute magnitude of MACHO 176.18833.411 lead to a distance of $(m-M)_{0}=14.29 \pm 0.13 \mathrm{mag}$.

In the OGLE-III catalog, Soszyński et al. (2011) marked $\sim 400$ stars as possessing proper motions relative to mean motion of the bulge that are large enough to be easily detected. MACHO 176.18833.411 is included in this list. To determine its proper motion, the centroids of stars were measured on each OGLE-IV (Udalski, Szymanski \& Szymanski 2015) image separately. All measured centroids were transformed to the common grid using positions of bright red giants i.e., stars belonging to the bulge, not the disk population. The transformed positions were fitted with a model that takes into account proper motion as well as differential refraction effects (see Poleski et al. 2013, for details).

With our proper motion, the space velocity and position vector can be resolved, and the $6 \mathrm{D}$ position and velocity information for MACHO 176.18833.411 is given in Table 1. For this convention, the Sun's orbital velocity vector $v_{\odot}=\left[U_{\odot}, V_{\odot}, W_{\odot}\right]=[14.0,12.24,7.25] \mathrm{km} \mathrm{s}^{-1}$, $V_{L S R}=220 \mathrm{~km} \mathrm{~s}^{-1}$ and position $=[8.28,0,0] \mathrm{kpc}$. The derived true space velocity is $-482 \pm 22 \mathrm{~km} \mathrm{~s}^{-1}$ relative to the Galactic rest frame.

\section{DISCUSSION}

4.1. Possible explanations for the high-velocity star 

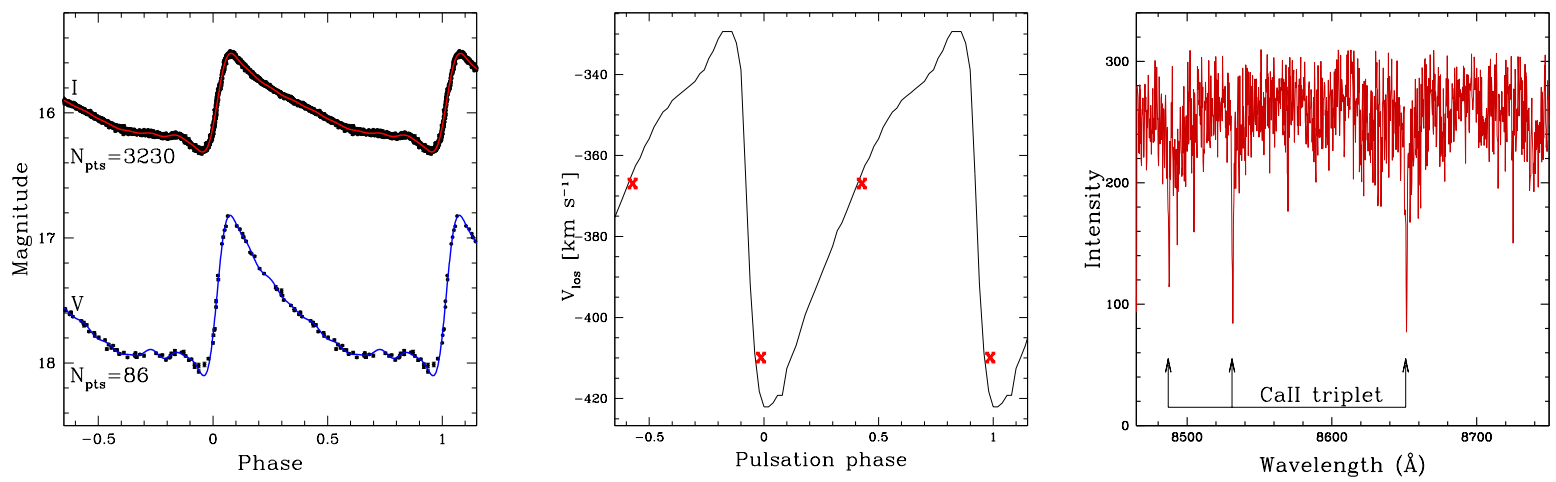

FIG. 1. - Left: Phased VI OGLE-IV light curves of MACHO 176.18833.411 with the Fourier fit over-plotted. Middle: Line-of-sight radial velocity versus pulsational phase for our two observations, over-plotted on a fundamental mode RRL radial velocity template and scaled by its $V$-amplitude (Liu 1991). Right: Wavelength-calibrated spectrum of MACHO 176.18833.411. The CaT lines are labeled for reference.

TABLE 1

Properties of MACHO 176.18833.411

\begin{tabular}{ll}
\hline \hline$\alpha(\mathrm{J} 2000)$ & $18: 00: 13.08$ \\
$\delta(\mathrm{J} 2000)$ & $-27: 15: 39.1$ \\
$l$ & 3.0795 \\
$b$ & -1.9209 \\
$<V>$ & $17.586 \mathrm{mag}$ \\
$<I>$ & $15.969 \mathrm{mag}$ \\
$V$-amp & $1.27 \pm 0.05 \mathrm{mag}$ \\
Period & $0.51521996 \pm 0.00000005 \mathrm{~d}$ \\
Time of max brightness & 2456000.21386 \\
{$[$ Fe/H] } & $-1.62 \pm 0.2($ from light curve $)$ \\
$(V-I)_{\text {min }}$ & $1.79 \pm 0.01$ \\
$\mathrm{E}(V-I)_{\min }$ & $1.21 \pm 0.03$ \\
$A_{I}$ & 1.49 \\
$(m-M)_{0}$ & $14.29 \pm 0.13 \mathrm{mag}$ \\
Heliocentric Distance & $7300 \pm 600 \mathrm{pc}^{-1}$ \\
Galactocentric Distance & $850 \mathrm{pc}$ \\
$V_{l o s}$ & $-372 \pm 8 \mathrm{~km} \mathrm{~s}^{-1}$ \\
$V_{G R V}$ & $-350 \pm 8 \mathrm{~km} \mathrm{~s}^{-1}$ \\
$\mu_{\alpha * \cos (\delta)}$ & $8.08 \pm 0.20 \pm 0.40 \mathrm{mas} \mathrm{yr}^{-1}$ \\
$\mu_{\delta}$ & $4.41 \pm 0.20 \pm 0.40 \mathrm{mas}^{-1}$ \\
$\mathrm{X}$ & $-1.04 \pm 0.56 \mathrm{kpc}^{-1}$ \\
$\mathrm{Y}$ & $0.39 \pm 0.03 \mathrm{kpc}$ \\
$\mathrm{Z}$ & $-0.243 \pm 0.02 \mathrm{kpc}^{-1}$ \\
$\mathrm{U}$ & $-377 \pm 20 \mathrm{~km} \mathrm{~s}^{-1}$ \\
$\mathrm{~V}$ & $-262 \pm 26 \mathrm{~km} \mathrm{~s}^{-1}$ \\
$\mathrm{~W}$ & $-147 \pm 20 \mathrm{~km} \mathrm{~s}^{-1}$
\end{tabular}

BRAVA-RR currently has surveyed only 94 RRLs, including MACHO 176.18833.411. Is such a bulge star therefore really anomalous? To give us some idea if this RRL in fact belongs to the bulge, we integrated its orbit through an assumed Galactic potential which is a sum of the potential of a logarithmic halo, Miyamoto-Nagai disk, and a Hernquist bulge, as in Hawkins et al. (2015). Uncertainties in the orbital integrations were estimated by a Monte Carlo approach, where the initial conditions were varied to within their uncertainties over 100 orbital integrations (see Hawkins et al. 2015, for details).

The orbital integration over the past 1 Gyr is shown in Figure 2, as is the distribution of the maximum distance from the Galactic plane, $\mathrm{Z}_{\max }$, and the minimum and maximum distance from the Galactic center for 100 orbital draws. Although MACHO 176.18833.411 is currently close to or in the bulge, its orbit clearly suggests it is not confined to the bulge. The escape velocity at the radius of the bulge is $\sim 650 \mathrm{~km} \mathrm{~s}^{-1}$; this means that a star with the high velocity of MACHO 176.18833.411 can still be inside the bulge. However, it is clear that MACHO 176.18833.411 spends most of its time well outside the radius of the formal bulge/bar structure.

We next address how likely it is for a bulge star to have such a negative radial velocity. The Galactocentic radial velocity, $V_{G R V}$, distribution of 229 giants toward the bulge at $(l, b)=(4,-2)$ and 320 RCGs toward the bulge at $(l, b)=(2.4,-2.2)$ is shown in Figure 3 The velocities of the giants are taken from APOGEE (the Apache Point Observatory Galactic Evolution Experiment), which is part of Sloan Digital Sky Survey III, and we analyse data from data release 12 (DR12, Alam et al. 2015). The velocities of the RCGs are taken from the GIRAFFE Inner Bulge Survey (GIBS, Zoccali et al. 2014). The mean velocity and velocity dispersion of both the APOGEE giant sample and the GIBS RCGs are within 1 sigma of the mean velocity and velocity dispersion for the RRLs in the $(l, b)=(3,-2.5)$ BRAVA-RR field (Kunder et al. 2016 , in prep). Assuming the radial velocity distribution for the giants, red clump and RRLs is Gaussian, a star with $V_{G R V}=-350 \mathrm{~km} \mathrm{~s}^{-1}$ is a four-sigma outlier in velocity space.

The 9000 BRAVA giant sample (Kunder et al. 2012), $\sim 17000$ ARGOS RCG sample (Freeman et al. 2013) and the 1200 Gaia-ESO Survey bulge RCG sample (RojasArriagada et al. 2014) probe further from the Galactic plane than where MACHO 176.18833 .411 resides, so more contamination from e.g., the disk and halo would be expected, but these large radial velocity surveys can also give an indication of how unusual it is for a star toward the bulge to have such a negative velocity. Within the Gaia-ESO Survey bulge sample, there is no star with $V_{G R V}$ less than $-350 \mathrm{~km} \mathrm{~s}^{-1}$ and $<0.1 \%$ of stars have $V_{G R V}$ less than $-300 \mathrm{~km} \mathrm{~s}^{-1}$. For both the BRAVA and ARGOS samples, $\sim 0.02 \%$ of stars have $V_{G R V}$ less than $-350 \mathrm{~km} \mathrm{~s}^{-1}$ and $\sim 0.1 \%$ of stars have $V_{G R V}$ less than $-300 \mathrm{~km} \mathrm{~s}^{-1}$.

\subsection{A halo star}

There are a few possibilities that can explain the presence of MACHO 176.18833.411. The most likely is that this star is a halo interloper that happens to be at the same distance and location as where bulge RRLs reside. This is supported by our orbital solution of MACHO 176.18833.411, which shows its $\mathrm{R}_{\max }$ is mostly 

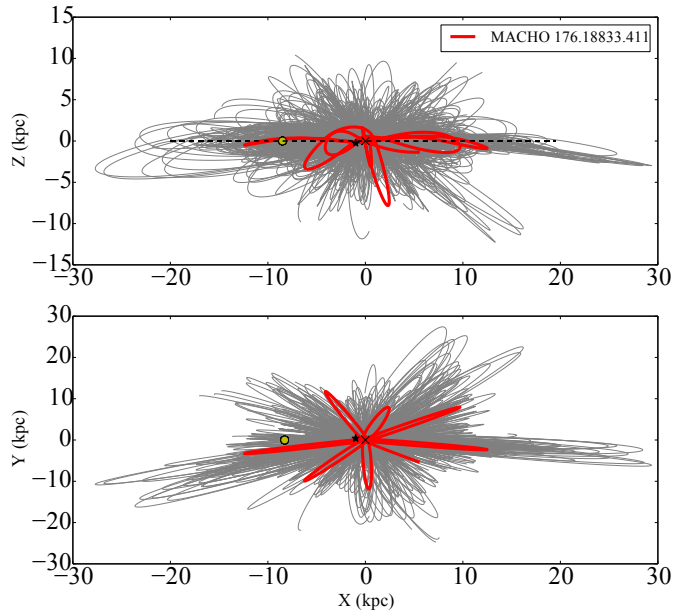
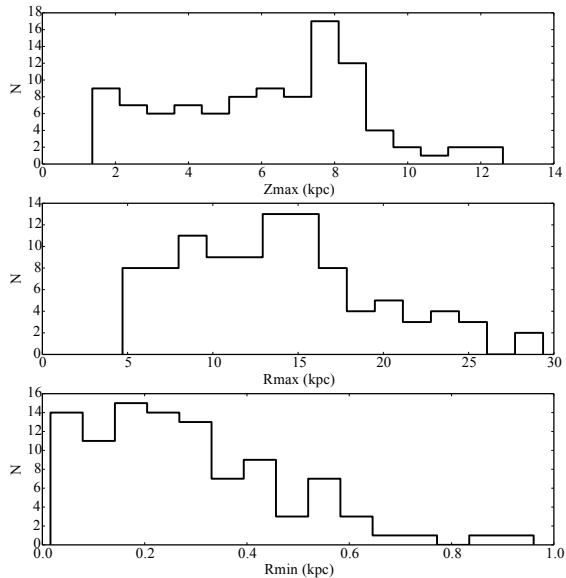

FIG. 2.- Left: A 1 Gyr orbital integration for MACHO 176.18833.411 (thick line). The open circle represents the Sun, the black 'x' represents the Galactic center and the black asterisk represents the current position of the RRL. The thin grey lines are 100 draws of the orbital integration to illustrate the uncertainty of the orbit. MACHO 176.18833.411 has an orbit consistent with that of the halo. Right: The distribution of $R_{\min }, R_{\max }$ and $Z_{\max }$ of the 100 orbital draws for our RRL.

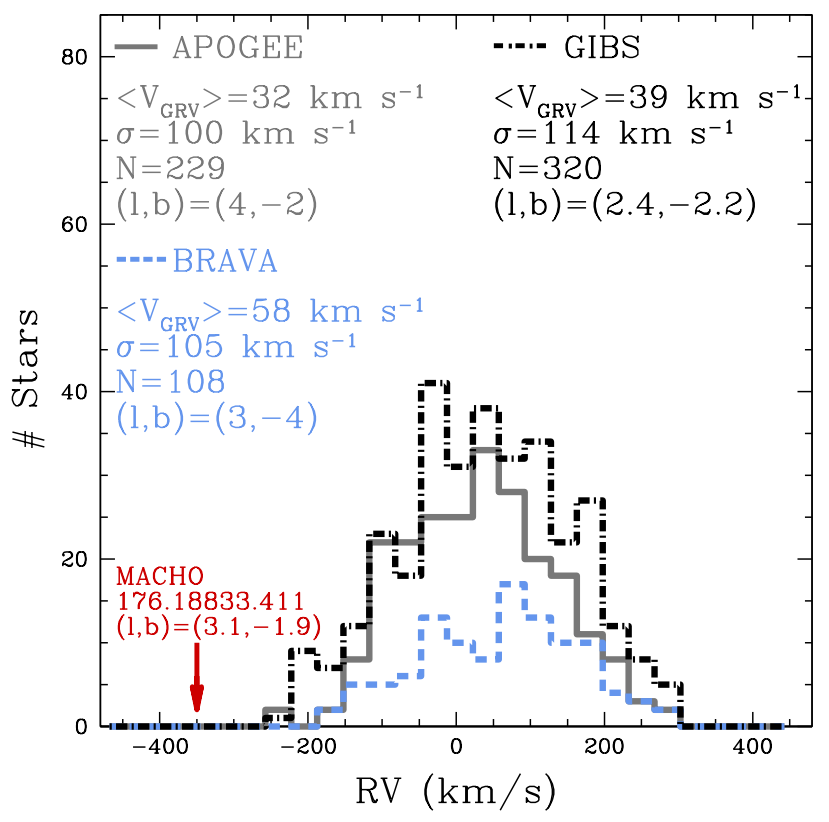

FIG. 3.- The Galactocentric velocity $\left(V_{G R V}\right)$ distribution of APOGEE giants (solid), BRAVA giants (dashed) and GIBS RCGs (dashed-dot) situated spatially close to MACHO 176.18833.411 (arrow). The mean GRV, dispersion of the $V_{G R V}$ and total number of stars are also given.

larger than $8 \mathrm{kpc}$, strongly suggesting this RRL is not confined to the bulge. The time spent in the bulge is $145 \pm 100 \mathrm{Myr}$, which corresponds to less than $15 \%$ of the integration. The $Z_{\max }$ of $\sim 8 \mathrm{kpc}$ suggests this star is not part of the disk, which is also consistent with its high velocity. This star is on an elliptical orbit (orbital ellipticity is 0.95 with a period of $\sim 140 \mathrm{Myr}$ ) and resembles a star in the halo.

Numerical simulations which match the kinematic observations of the bulge well do not predict such high velocity bulge stars in this region of the sky (Shen et al. 2010), although we note that it is possible for significant radial velocity outliers to be present in any popula- tion. Instead, high velocity RRLs have been found in the halo (e.g., $\sim 1.5 \%$ of the local RRLs in the Layden 1994 and Kollmeier et al. 2013 samples have $V_{G R V}$ less than $-300 \mathrm{~km} \mathrm{~s}^{-1}$, which is more than a factor of 10 larger than high velocity giants and RCGs found in the bulge surveys discussed previously). Similarly within the halo globular cluster (GC) sample, $3 \%$ have $V_{G R V}$ less than $-350 \mathrm{~km} \mathrm{~s}^{-1}$.

If this RRL is a halo interloper, we presume additional halo contamination exists in the bulge RRL sample, with velocities indistinguishable from bulge stars. We would then expect at least a few percent of the RRLs located in the direction toward the bulge to be halo constituents. To obtain a rough approximation of the presence of the halo, the relation of the RRL density profile in the halo is extrapolated to the Galactic center (Watkins et al. 2009) as shown in Figure 4. To find the total number of halo RRLs expected in our observations, we use this relation along with the following approximations: (1) the bulge is a cylinder with a radius of $2 \mathrm{kpc}$ and a minor-tomajor axial ratio of 0.55 , and (2) the bulge extends from $l=-10$ to $l=+10$ and $b=-10$ to $b=+10$ degrees, and so the AAOmega 3 square degree field covers $3 / 314$ of the Galactic bulge. It follows, then, that $\sim 85 \pm 70$ halo RRLs are expected with a Galactocentric distance of $0.9 \mathrm{kpc}$ in a 3 square degree field. The uncertainty in the number of halo RRLs is a function of the exact scale height and length of the bulge, and is illustrated in Figure 4. The total number of OGLE RRLs in our 3 square degree field is $\sim 1400$, and the Galactocentric distance of these stars peaks at $0.8 \mathrm{kpc}$. Therefore, assuming the OGLE sample of RRLs is relatively complete, $\sim 6 \%$ of the OGLE RRLs stars toward $(l, b)=(3,-2.5)$ are expected to be from the halo, and it is not unexpected for a few percent of halo stars to exist so close, both in spatial and in distance distribution, to the Galactic center. Proper motions of a larger sample of RRLs would be desirable to identify halo RRLs from bulge RRLs.

It is worth noting that the orbit of MACHO 176.18833.411 has its largest excursions perpendicular to the plane - this extreme high velocity star 


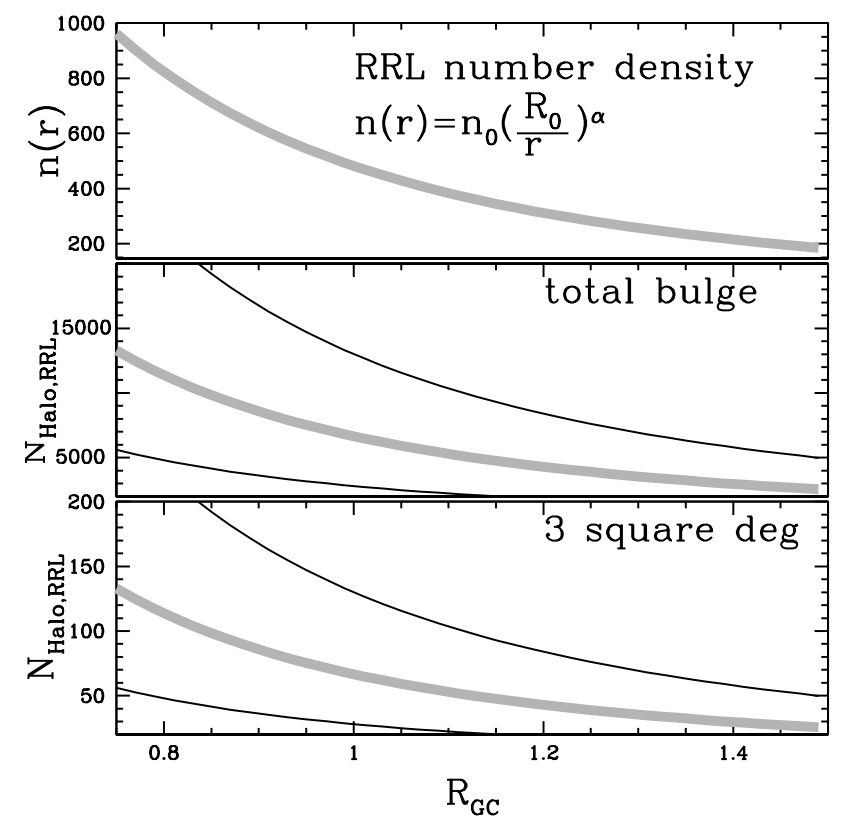

FIG. 4.- Top: The RRL number density as a function of galactocentric distance, $R_{G C}$, in the $0.75-1.5 \mathrm{kpc}$ range using a power law derived from SDSS Stripe $82 \mathrm{RR}$ Lyrae stars (Watkins et al. $2009)$, where $\left(\mathrm{n}_{0}, \mathrm{R}_{0}, \alpha\right)=\left(0.26 \mathrm{kpc}^{-3}, 23 \mathrm{kpc}, 2.4\right)$. Middle: The

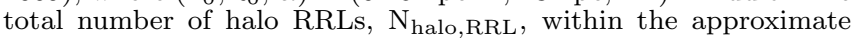
bulge volume as a function $R_{G C}$. The thick grey lines indicates the $N_{\text {halo, } R R L}$ assuming a bulge radius of $2 \mathrm{kpc}$ and a minor to major axis ratio of 0.55 ; the thin black lines indicate $\mathrm{N}_{\text {halo, RRL }}$ assuming a bulge radius of 1.5 and $2.5 \mathrm{kpc}$. Bottom: Same as the middle panel, for for a 3 square degree bulge field.

is confined (statistically) vertically. If the most extreme halo stars have an almost spheroidal zone of excursion, the (halo) population from where this star originated from is not the most extreme. It may well be that this RRL is a relic from an earlier era, but likely not the earliest.

\subsection{A bulge star}

A second possibility is that MACHO 176.18833.411 is on the extreme edge of the bulge RRL radial velocity distribution. This could be in line with the notion discussed by Dékány et al. (2013), that the bulge RRLs follow a spheroidal, centrally concentrated distribution, as then eccentric orbits and therefore stars with large radial velocities, would be expected. If the bulge RRLs follow a Gaussian radial velocity distribution with a $\sigma=200 \mathrm{~km} \mathrm{~s}^{-1}$, it would be expected to find a $V_{G R V}=-350 \mathrm{~km} \mathrm{~s}^{-1}$ radial velocity star. Such a large velocity distribution is not observed for the 94 BRAVA-RR stars in this area of the sky, but although a Gaussian distribution is convenient, it is often wrong out on the tails, in a sense that the tails are too small. It has also been shown that multiple RRLs populations exist in the bulge (Pietrukowicz et al. 2014), and so there may be a small population of RRLs more kinematically hot than the majority of bulge RRLs, and that MACHO 176.18833.411 is part of this more kinematically hot component.

Another motivation for MACHO 176.18833.411 being a member of the bulge RRL population is that a similar proportion of high negative velocity stars are found in bulge stars exhibiting maser emissions (in both $\mathrm{OH} / \mathrm{IR}$ and $\mathrm{SiO}$ masers, van Langevelde et al. 1992 Fujii et al. 2006). However, it is unclear why these mass-losing infrared objects, typically Asymptotic Giant Branch (AGB) stars, which are thought to be much younger than RRLs, (Mouhcine \& Lançon 2002, 1 to a few Gyr, ), would be more dynamically similar to bulge RRLs than red giants or RCGs.

\subsection{A runaway star}

A third scenario to explain the velocity of MACHO 176.18833.411 is that it was ejected from the Galactic center due to an interaction - e.g., a star-binary or a star - black hole collision, or ejected out of a globular cluster. It is unlikely that this star is a hypervelocity star (HVS), as its velocity is still well within the realm of being bound to the Milky Way (e.g., Kenyon et al. 2008) MACHO 176.18833 .411 may be a runaway star, though. Evolved stars have been shown to be runaways (Kilic et al. 2013), as have horizontal branch stars (Pereira et al. 2013), although by far the largest population of runaway stars currently known are younger, more massive stars (e.g. Bromley et al. 2009). Runaway stars are expected to dominate at low Galactic latitudes and are located preferentially in the direction of the Galactic center, between $l=325-35$ (e.g., Bromley et al. 2009). The expected metallicity of runaways is $\sim[\mathrm{Fe} / \mathrm{H}] \sim-1.5 \mathrm{dex}$, slightly more metal-rich than the halo and comparable to MACHO 176.18833.411.

\section{CONCLUSIONS}

In this paper, we take a detailed look at a high negative-velocity RRL observed toward the Galactic bulge found in the BRAVA-RR survey. Stars of such high velocity are rare among bulge giants, and the more precise distance of the RRL makes it possible to explore its origin in greater detail, by integrating its orbit. We argue that MACHO 176.18833 .411 is most likely a halo interloper, suggesting that contamination from halo stars is relevant when attempting to trace out the metal-poor tail of the bulge's metallicity distribution function (e.g., Howes et al. 2014).

We thank the Australian Astronomical Observatory, which have made these observations possible. This research was supported in part by the National Science Foundation under Grant No. NSF PHY11-25915. This work was supported by Sonderforschungsbereich SFB 881 "The Milky Way System" (subprojects A4, A5, A8) of the German Research Foundation (DFG). This work has been partially supported by the Polish Ministry of Science and Higher Education through the program "Ideas Plus" award No. IdP2012 000162 to IS. RMR acknowledges support from grant AST-1413755 from the National Science Foundation. C.I.J. gratefully acknowledges support from the Clay Fellowship, administered by the Smithsonian Astrophysical Observatory.

\section{REFERENCES}

Alam, S., Albareti, F.D., Allende Prieto, C. et al. 2015, ApJS, submitted (arXiv:150100963)
Benedict, G. F., McArthur, B. E., \& Feast, M. W. et al. 2011, AJ, 142, 187 
Bono, G., Caputo, F., di Criscienzo, M. 2007, A\&A, 476, 779

Brown, W.R. Anderson, J., Gnedin, O.Y., Bond, H.E., Geller, M.J., Kenyon, S.J. 2015, ApJ, 804, 49

Bromley, B.C., Kenyon, S.J., Brown, W.R. \& Geller, M.J. 2009, ApJ, 706, 925

Carretta, E., Bragaglia, A., Gratton, R., D’Orazi, V. \& Lucatello, S. 2009, A\&A, 508, 695

Catelan, M. \& Cortés, C. 2008, ApJ, 676, L135

Dékány, I., Minniti, D., Catelan, M., Zoccali, M., Saito, R. K. Hempel, M. \& Gonzalez, O. A. 2013, ApJ, 776, 19

Freeman, K., Ness, M., Wylie-de-Boer, E., Athanassoula, E., Bland-Hawthorn, J., Asplund, M., Lewis, G., Yong, D., Lane, R., Kiss, L. \& Ibata, R. 2013, MNRAS, 428, 3660

Fujii, T., Deguchi, S., Ita, Y., Izumiura, H., Kameya, O., Miyazaki, A. \& Nakada, Y. 2006, PASJ, 58, 529

Gonzalez, O. A., Rejkuba, M., Zoccali, M., Valenti, E., Minniti, D., Schultheis, M., Tobar, R. \& Chen, B. 2012, A\&A, 543, 13

Gratton, R.G. 1987, MNRAS, 224, 175

Groenewegen, M.A.T. \& Blommaert, J.A.D.L. 2005, A\&A, 443, 143

Guldenschuh, K. A. et al. 2005, PASP, 117, 721

Hawkins, K., Kordopatis, G., Gilmore, G. et al. 2015, MNRAS, 447,2046

Howes, L.M., Asplund, M., Casey, A.R. et al. 2015, MNRAS, 445, 4241

Johnson, C.I., Rich, R.M., Kobayashi, C., Kunder, A., Pilachowski, C., Koch, A. \& De Propris, R. 2013, ApJ, 765, 157

Jurcsik, J. \& Kovács, G. 1996, A\&A, 312, 111

Kazantzidis, S., Bullock, J. S., Zentner, A. R., Kravtsov, A. V., \& Moustakas, L. A. 2008, ApJ, 688, 254

Kenyon, S.J., Bromley, B.C., Geller, M.J. \& Brown, W.R. 2008, ApJ, 680, 312

Kilic, M., Gianninas, A., Brown, W.R. 2013, MNRAS, 434, 3582

Kollmeier, J.A., Szczygiel, D.M., Burns, C.R. 2013, ApJ, 775, 57

Kovács, G. 2005, A\&A, 438, 227

Kunder, A. \& Chaboyer B. 2008, AJ, 136, 2441
Kunder, A., Popowski, P.; Cook K.H. \& Chaboyer B. 2008, AJ, 135,631

Kunder, A., Koch, A., Rich, R. M., et al. 2012, AJ, 143, 57

Layden, A. 1994, AJ, 108, 1016

Lee, Y.-W. 1992, AJ, 104, 1780

Liu, T. 1991, PASP, 103, 205

Mouhcine, M., \& Lançon, A. 2002, A\&A, 393, 149

Nataf, D.M., Gould, A., Fouqué, P. et al. 2013, ApJ, 769, 88

Pereira, C.B., Jilinski, E.G., Drake, N.A., Ortega, V.G. \& Roig, F. 2013, A\&A, 559, 12

Pietrukowicz et al. 2014, ApJ, submitted

Poleski, R., Udalski, A., Gould, A., Szymański, M. K., Soszyński, I., Kubiak, M., Pietrzyński, G., Ulaczyk, K. \& Wyrzykowski, Ł. 2013, ApJ, 776, 76

Preston, G. 2011, AJ, 141, 6

Rojas-Arriagada, A., Recio-Blanco, A., Hill, V. et al. 2014, A\&A, 569,103

Saha, K. \& Gerhard, O. 2013, MNRAS, 430, 2039

Shen, J., Rich, R. M., Kormendy, J., et al. 2010, ApJ, 720, L72

Soszyński, I., Dziembowski, W. A., Udalski, A., Poleski, R., Szymański, M. K., Kubiak, M., Pietrzyński, G., Wyrzykowski, Ł., Ulaczyk, K., Kozłowski, S. \& Pietrukowicz, P. 2011, AcA, 61,1

Soszyński, I., Udalski, A., Szymański, M. K. et al. 2014, AcA, 64, 177

Stanek, K. Z., Mateo, M., Udalski, A., Szymański, M., Kaluzny, J. \& Kubiak M., 1994, ApJ, 429, L73

Udalski, A., Szymański, M. K. \& Szymański, G. 2015, AcA, 65, 1 van Langevelde, H. J., Brown, A. G. A., Lindqvist, M., Habing,

H. J., \& de Zeeuw, P. T. 1992, A\&A, 261, 17

Walker, A.R. \& Terndrup, D.M. 1991, ApJ, 378, 119

Watkins L. L., Evans, N.W., Belokurov, V. et al., 2009, MNRAS, 398,1757

Wegg, C. \& Gerhard, O. 2013, MNRAS, 435, 1874 